\title{
Long non-coding RNA FOXD2-AS1 plays an oncogenic role in hepatocellular carcinoma by targeting miR-206
}

\author{
YUANHONG CHANG ${ }^{1,2}$, JIE ZHANG $^{3}$, CANCAN ZHOU $^{4}$, GUANGLIN QIU $^{5}$, GUANGHUI WANG ${ }^{5}$, \\ SHUFENG WANG ${ }^{5}, \mathrm{XINMING} \mathrm{CHANG}^{1}, \mathrm{XUQI} \mathrm{LI}^{5}$ and $\mathrm{LIN} \mathrm{FAN}^{5}$ \\ ${ }^{1}$ Department of Gastroenterology, The First Affiliated Hospital of Xi'an Jiaotong University, Xi'an, \\ Shaanxi 710061; ${ }^{2}$ Department of Gastroenterology, Xi'an No. 4 Hospital, The Affiliated Guangren Hospital, \\ School of Medicine, Xi'an Jiaotong University, Xi'an, Shaanxi 710004; ${ }^{3}$ First Department of General Surgery, \\ Shaanxi Provincial Tumor Hospital, Xi'an, Shaanxi 710068; Departments of ${ }^{4}$ Hepatobiliary Surgery and \\ ${ }^{5}$ General Surgery, The First Affiliated Hospital of Xi'an Jiaotong University, Xi'an, Shaanxi 710061, P.R. China
}

Received May 5, 2018; Accepted September 26, 2018

DOI: $10.3892 / o r .2018 .6752$

\begin{abstract}
Recently, long non-coding RNA (lncRNA) FOXD2 adjacent opposite strand RNA 1 (FOXD2-AS1) has been recognized to function as an oncogene in several human tumors, and FOXD2-AS1 dysregulation has been closely associated with carcinogenesis and tumor progression. Nevertheless, the correlation between the aberrant expression of FOXD2-AS1 and the prognosis of hepatocellular carcinoma (HCC) has not yet been elucidated. In the present study, FOXD2-AS1 was found to be overexpressed in HCC tissues, and FOXD2-AS1 overexpression resulted in significantly shortened patient survival. FOXD2-AS1 overexpression enhanced the viability and metastasis of HCC cells in vitro and in vivo, as revealed by MTT, wound healing and cell migration assays. In addition, mechanistic studies revealed that FOXD2-AS1 upregulated the expression of the miR-206 target gene annexin A2 (ANXA2) by acting as a miR-206 sponge. In summary, FOXD2-AS1 was concluded to function as an oncogene in HCC and to upregulate ANXA2 expression in part by 'sponging' miR-206.
\end{abstract}

\section{Introduction}

Hepatocellular carcinoma (HCC) is clinically considered a lethal cancer that usually results in intra- and extrahepatic metastasis and has a poor prognosis. Although a majority of HCC cases occur in underdeveloped nations, HCC is the most frequent cancer as well as the second leading cause of mortality in China (1). Although earlier studies have revealed

Correspondence to: Dr Xuqi Li or Dr Lin Fan, Department of General Surgery, The First Affiliated Hospital of Xi'an Jiaotong University, 277 West Yanta Road, Xi'an, Shaanxi 710061, P.R. China E-mail: lixuqi@163.com

E-mail: linnet@mail.xjtu.edu.cn

Key words: long non-coding RNA, FOXD2-AS1, microRNA, hepatocellular carcinoma, miR-206 links between several genes and HCC, the underlying mechanisms remain unexplored. Therefore, an understanding of the underlying pathophysiological factors associated with HCC is vital for uncovering novel prognostic biomarkers and developing therapeutic strategies.

The transcription of RNA from non-protein-coding segments of DNA is one of the most crucial discoveries of the postgenomic era (2). Protein-coding genes constitute merely $\sim 2 \%$ of the human genome, while a greater proportion of the genome includes non-coding RNAs (ncRNAs) such as long ncRNAs (lncRNAs). Recent research has revealed the roles of lncRNAs in cancer; IncRNAs can act either as oncogenes or as tumor suppressors. Several research groups have established that metastasis-associated lung adenocarcinoma transcript 1 (MALAT1) positively modulates growth, apoptosis, and migration in many cancers $(3,4)$. On the other hand, maternally expressed gene 3 (MEG3) was revealed to function as a tumor inhibitor that promoted p53-mediated transactivation $(5,6)$. IncRNA activated by transforming growth factor $\beta$ (TGF- $\beta$ ) (lncRNA-ATB), which is an important regulator of the invasion-metastasis cascade, was revealed to promote cell invasion through its role as a competing endogenous RNA (ceRNA) for zinc finger E-box-binding homeobox (ZEB) genes and facilitated the colonization of disseminated HCC cells in distant organs by binding to interleukin-11 (IL-11) mRNA (7). A previous study found that FOXD2 adjacent opposite strand RNA 1 (FOXD2-AS1) regulated tumor development in lung cancer (8). In terms of microRNAs (miRNAs), endogenous transcripts containing miRNA response elements (MREs) have been proposed to interact with each other by acting as miRNA sponges or as ceRNAs, thus forming large-scale regulatory networks across the transcriptome (9). For example, Tan et al demonstrated that the double-negative feedback loop between IncRNA taurine upregulated 1 (TUG1) and miR-145 promoted epithelial to mesenchymal transition and radioresistance in human bladder cancer cells (10). Nevertheless, the relationship between aberrant FOXD2-AS1 expression and malignant behavior in HCC remains unclear, and the mechanism underlying the oncogenic activity of FOXD2-AS1 warrants elucidation. 
We hypothesized that IncRNA FOXD2-AS1 contributes to HCC progression by acting as a miRNA sponge and, moreover, that FOXD2-AS1 expression is increased in HCC tissues and is correlated with inferior prognosis. Additional investigations revealed that when overexpressed, FOXD2-AS1 functioned like a ceRNA that enhanced cell viability and migration, thus resulting in the upregulation of the annexin A2 (ANXA2) protein, which was regulated by the targeting of miR-206 by FOXD2-AS1. As a whole, this study established that the upregulation of FOXD2-AS1 promoted the viability of HCC cells in part through the role of FOXD2-AS1 as a miR-206 sponge.

\section{Materials and methods}

Patients. During 2008-2010, a total of 140 HCC tissues and the corresponding neighboring healthy hepatic tissues from patients subjected to resection were acquired from the First Affiliated Hospital of Xi'an Jiaotong University (Xi'an, China). The patients were monitored via phone calls or visits every 6 months until their death or the completion of the study. The patients had not undergone chemotherapy or radiotherapy prior to surgery, and the tissue samples were subjected to pathological examination. Prior to the the study, the patients signed written informed consent forms. In addition, the present study was approved by the Ethics Committee of the First Affiliated Hospital of Xi'an Jiaotong University (Xi'an, China).

Cell culture, cell transfection and MTT assay. Human liver cancer cell lines (Hep3B, MHCC97-L, MHCC97-H, SK-HEP1 and HCCLM3) and a cultured normal liver cell line (HL7702) were obtained from the Cell Bank of the Chinese Academy of Sciences. These cells were cultured in RPMI-1640 medium containing 10\% fetal bovine serum (FBS; Gibco; Thermo Fisher Scientific, Inc. Waltham, MA, USA) at $37^{\circ} \mathrm{C}$ in $5 \% \mathrm{CO}_{2}$. Cells in the logarithmic growth phase were monitored after being passaged every 2-3 days. The miRNA mimics, inhibitors and small interfering RNAs (siRNAs) were obtained from GenePharma Co., Ltd. (Shanghai, China). Oligonucleotide and plasmid transfections were carried out with Lipofectamine ${ }^{\mathrm{TM}} 2000$ transfection reagent (Invitrogen; Thermo Fisher Scientific, Inc.), according to the manufacturer's instructions. Following transfection, the cells were collected and used for subsequent studies. The siRNA oligo sequences were as follows: siFOXD2-AS1 sense, 5'-GCGCG GUUGUUGAGACCAAGG-3' and siFOXD2-AS1 antisense, 5'-UUGGUCUCAACAACCGCGCAG-3'.

After transfection, cell proliferation was analyzed via a 3-(4,5-dimethyl-2-thiazolyl)-2,5-diphenyl-2- $H$-tetrazolium bromide (MTT) assay (Promega Corp., Madison, WI, USA) according to the manufacturer's instructions. Cells $\left(5 \times 10^{3}\right.$ cells/ well) were seeded in 96-well plates and treated $24 \mathrm{~h}$ later. After a further $24 \mathrm{~h}, 20 \mu \mathrm{l}$ of $5 \mathrm{mg} / \mathrm{ml}$ MTT was added, and the plates were placed in an incubator for $4 \mathrm{~h}$. Next, the supernatant was aspirated and $200 \mu \mathrm{l}$ of dimethyl sulfoxide (DMSO) was added to the wells to dissolve the formazan. The optical density (OD) was determined at $450 \mathrm{~nm}$.

RNA isolation and quantitative real-time PCR ( $q R T-P C R)$. RNA was extracted with TRIzol reagent (Invitrogen;
Thermo Fisher Scientific, Inc.). After RNA extraction, first strand cDNA was synthesized with a High Capacity cDNA Reverse Transcription kit (Applied Biosystems; Thermo Fisher Scientific, Inc.). The mRNA and lncRNA expression analyses were carried out by RT-PCR (11). qRT-PCR was carried out with SYBR ${ }^{\circledR}$ Premix Ex Taq ${ }^{\mathrm{TM}}$ II (Takara Biotechnology Co., Ltd., Dalian, China) on a StepOne Plus Real-Time PCR System (Applied Biosystems; Thermo Fisher Scientific, Inc.) following the supplier's mRNA analysis protocols. GAPDH and U6 were used as the endogenous controls for lncRNAs and miRNAs, respectively. The qRT-PCR experiments were carried out in triplicate, whereas the changes in the expression of the candidate genes were analyzed via the $2^{-\Delta \Delta \mathrm{Cq}}$ method (12). The primer sequences were as follows: FOXD2-AS1 forward, 5'-TGTTCGTGGGAAGAGGGTTG-3' and reverse, 5'-TACCA CTCCGGGAACTCTGT-3'; and GAPDH forward, 5'-ACTGC CACCCAGAAGACT-3' and reverse, 5'-GCTCAGTGTAGCC CAGGAT-3'. qRT-PCR included an initial denaturation cycle at $95^{\circ} \mathrm{C}$ for $2 \mathrm{~min}$, followed by 35 cycles of denaturation at $98^{\circ} \mathrm{C}$ for $10 \mathrm{sec}$ and annealing and extension at $60^{\circ} \mathrm{C}$ for $45 \mathrm{sec}$.

Vector construction and transfection. Full-length lncRNA FOXD2-AS1 was amplified using Phusion Flash High-Fidelity PCR Master Mix (Thermo Fisher Scientific, Inc.) and cloned into pcDNA3.1 (Invitrogen; Thermo Fisher Scientific, Inc.); the resulting vector was named pcDNA3.1-FOXD2-AS1. FOXD2-AS1 constructs mutated at the putative miR-206 target site were generated with the appropriate primers. The resulting vectors were sequenced and were named FOXD2-AS1-WT (wild-type FOXD2-AS1) and FOXD2-AS1-Mut (mutated FOXD2-AS1). siRNA targeting ANXA2 was obtained from GenePharma Co., Ltd. The double-stranded miRNA mimic (miR-206) and the control miRNA (miR-con) were obtained from GenePharma Co., Ltd. The vectors and miRNAs were transfected into HCC cells using Lipofectamine 3000 (Invitrogen; Thermo Fisher Scientific, Inc.). The primers used for cloning and plasmid assembly were as follows: pCDNA/FOXD2-AS1-F, 5'-GGATCCCCTGTTCGGCGT CTTGCAGCAGTGC-3') and pCDNA/FOXD2-AS1-R, 5'-CTC GAGGGAATGAATAACTTCAGTC-3'.

Wound healing assay. The wound healing assay was carried out to verify cell metastasis. First, cells $\left(5 \times 10^{5}\right.$ cells/well $)$ suspended in DMEM were cultured in 6-well plates as confluent monolayers for $24 \mathrm{~h}$. Subsequently, scratches were made on the monolayer surface with a $200-\mu 1$ pipette tip. Images depicting cell migration across the scratches were captured in 5 randomly selected fields at 0 and $24 \mathrm{~h}$ following treatment.

Cell migration assay. To study the impact of lncRNA FOXD2-AS1 on cell migration, Transwell assays were performed with $8-\mu \mathrm{m}$ pore chambers (BD Biosciences, Franklin Lakes, NJ, USA). For the Transwell assays, cells in which lncRNA FOXD2-AS1 was either overexpressed or knocked down were added to serum-free medium and permitted to migrate in the direction of medium supplemented with $10 \% \mathrm{FBS}$ at $37^{\circ} \mathrm{C}$ for $24 \mathrm{~h}$. The migrated cells were treated with $100 \%$ methanol for $30 \mathrm{~min}$, whereas the nonmigrated cells were removed. Subsequently, the cells on the 
underside of the membrane were stained with $0.1 \%$ crystal violet for $20 \mathrm{~min}$. The number of cells in 5 arbitrary fields of each replicate was determined with a light microscope (Nikon ECLIPSE 80i; x100 magnification). All experiments were repeated three times.

RNA immunoprecipitation (RIP) assay. Hep3B cells were used in the RIP assay along with an anti-Ago2 antibody (Cell Signaling Technology, Inc., Danvers, MA, USA) and a Magna RIP ${ }^{\mathrm{TM}}$ RNA-Binding Protein Immunoprecipitation kit (EMD Millipore, Billerica, MA, USA). After antibody recovery with protein A/G beads, qRT-PCR was carried out to quantify FOXD2-AS1 and the target miRNAs in the precipitate.

Bioinformatics analysis. The putative miRNA binding sites on lncRNA FOXD2-AS1 were identified by DIANA software (http:/carolina.imis.athena-innovation.gr/diana_tools/ web/index.php) with the minimum cutoff score set at 0.802 .

Luciferase reporter assay. The $3^{\prime}$ untranslated regions (3'-UTRs) of FOXD2-AS1-WT and FOXD2-AS1-Mut were cloned into the pGL3-basic luciferase reporter vector (Promega Corp.). For the luciferase reporter assay, $100 \mathrm{ng}$ of either the FOXD2-AS1-WT vector or the FOXD2-AS1-Mut vector, along with $100 \mathrm{nM}$ miR-206 mimic and $20 \mathrm{ng}$ of a Renilla luciferase vector (Promega Corp.) as the control, were transfected into cells by Lipofectamine 3000 (Invitrogen; Thermo Fisher Scientific, Inc.). The relative luciferase activity was determined via a Dual-Luciferase reporter gene assay system by normalization to the Renilla luciferase activity at $48 \mathrm{~h}$ after transfection. Transfections were carried out in duplicate and repeated three times.

Western blot analysis. Cell lysis was carried out in RIPA buffer supplemented with a protease inhibitor (Roche Diagnostics, Indianapolis, IN, USA) and heated at $95^{\circ} \mathrm{C}$ for $5 \mathrm{~min}$. The protein concentration was quantified with a BCA Protein Assay kit (Qiagen, Valencia, CA, USA), according to the manufacturer's protocol. Lysates (40 $\mu \mathrm{g}$ protein) were purified by $10 \%$ SDS-PAGE gels and transferred to nitrocellulose membranes over a subsequent 2-h time period. Membranes were blocked in 5\% skim milk in 1X TBST for $2 \mathrm{~h}$ at room temperature and incubated with ANXA2 (1:1,000 dilution; cat. no. 8235; Cell Signaling Technology, Inc.) and GAPDH (1:2,000 dilution; cat. no. 5174; Cell Signaling Technology, Inc.) primary antibodies at $4^{\circ} \mathrm{C}$ overnight. Next, the membranes were rinsed three times with $1 \%$ TBST, incubated with secondary antibodies (goat anti-rabbit IgG-HRP;0 1:10,000 dilution; cat. no. ab6721; Abcam, Cambridge, MA, USA) at room temperature for $1 \mathrm{~h}$ and detected with enhanced chemiluminescence (ECL) reagents (Pierce; Thermo Fisher Scientific, Inc.). In addition, densitometry was employed to quantify the intensity of the protein bands (Image Lab software 4.1; Bio-Rad Laboatories, Inc., Hercules, CA, USA) and normalize them to their respective GAPDH bands.

Xenograft tumor model. Twelve 4- to 6-week-old female BALB/c nude mice were obtained from Shanghai SLAC Laboratory Animal Co., Ltd. and maintained in a sterile pathogen-free environment. The specific housing conditions were as follows: Temperature, $21 \pm 2^{\circ} \mathrm{C}$; humidity, $30-70 \%$; 12 -h light/dark cycle; the ingested food and water were sterile feed and sterilized bottled water. Animals were fed ad libitum mouse chow and given free access to water. All animal experiments were performed with stringent adherence to the Guide for the Care and Use of Laboratory Animals and were approved by the Laboratory Animal Care Committee of the Xi'an Jiaotong University (no. XJTULAC2018-462). We used 6 mice per group. Control (LV-CON) and FOXD2-AS1-overexpressing (LV-FOXD2-AS1) HCC cells were trypsinized and harvested in serum-free DMEM; $0.1 \mathrm{ml}$ serum-free DMEM containing $3 \times 10^{6}$ cells was then administered by subcutaneous injection into the right flank of the mice. Tumor growth was evaluated by determining tumor diameters with a digital caliper, whereas the tumor volume was determined using the following formula: tumor volume $=a b^{2} / 2$, where $a$ is the larger and $b$ is the smaller of the two dimensions. According to the Institutional Animal Care and Use Committee guidelines, the maximum allowable tumor size for mice is $4.2 \mathrm{~cm}^{3}$. The mice were sacrificed via $\mathrm{CO}_{2}$ inhalation 24 days after injection, and the tumors were harvested by excision for further analysis.

Statistical analysis. Differences between the groups were determined by one way ANOVA followed by LSD post hoc test, the Wilcoxon signed-rank test, or Pearson's $\chi^{2}$ test as appropriate. Differences were regarded as statistically significant for P-values $<0.05$. Pearson correlation analysis was used to examine the association between FOXD2-AS1 expression and miR-206. Overall survival (OS) curves were estimated using the Kaplan-Meier method and the log-rank test. Cox proportional hazard models were used, and P-values were determined by SPSS 20.0 software (IBM Corp., Armonk, NY, USA).

\section{Results}

FOXD2-AS1 expression is increased in HCC and is linked to inferior prognosis. To assess the involvement of FOXD2-AS1 in oncogenesis, we initially examined FOXD2-AS1 expression levels in $360 \mathrm{HCC}$ tissue specimens in TCGA. To determine the correlation between FOXD2-AS1 expression and clinicopathological characteristics, the FOXD2-AS1 expression levels were classified as low $(n=252)$ or high $(n=108)$ according to the Youden index (Fig. 1A). Then, we analyzed the OS curves to determine whether the FOXD2-AS1 expression level was related to prognosis. As shown in Fig. 1B, high FOXD2-AS1 expression in HCC tissues was linked to shorter OS $(\mathrm{P}=0.0070$, log-rank test). Additionally, we determined the levels of FOXD2-AS1 in six HCC cell lines (Hep3B, MHCC97-L, MHCC97-H, SK-HEP1 and HCCLM3); FOXD2-AS1 expression was considerably higher in all six HCC cell lines than in the normal human liver cell line HL7702 (Fig. 1C). Of the six HCC cell lines, SK-HEP1 and HCCLM3 cells had the highest FOXD2-AS1 expression, while Hep3B exhibited the lowest FOXD2-AS1 expression. To explore whether FOXD2-AS1 is detectable in HCC, we studied its expression in $140 \mathrm{HCC}$ tissues and neighboring healthy hepatic tissues. As shown in Fig. 1D, greater expression of FOXD2-AS1 was observed in tumor tissues than in the neighboring healthy tissues $(\mathrm{P}<0.01)$. These results indicated that FOXD2-AS1 likely plays a key role in tumor progression in human HCC. 

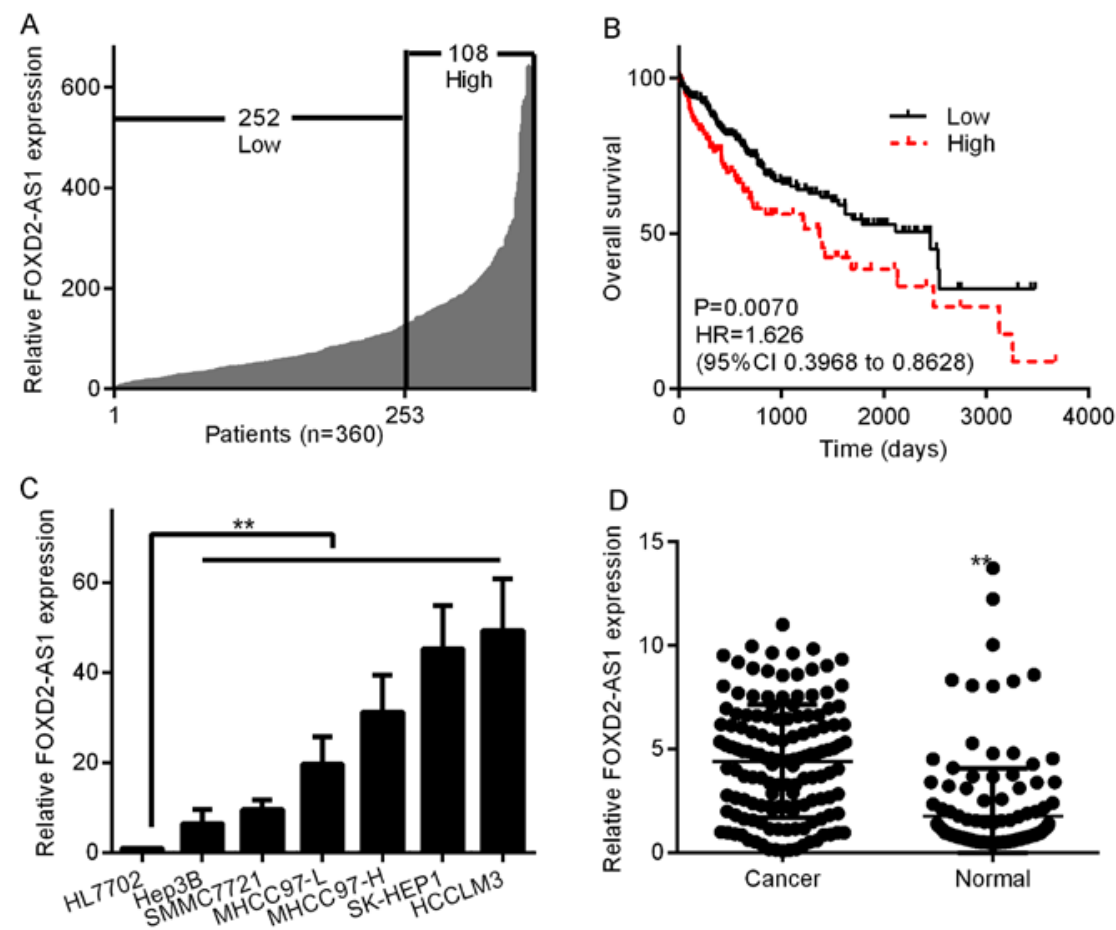

Figure 1. Expression of FOXD2-AS1 in HCC and its relation to the prognosis of patients. (A) A total of 360 recruited HCC patients were separated into low $(n=252)$ or high $(n=108)$ FOXD2-AS1 expression groups according to the Youden index. (B) OS curves were analyzed by FOXD2-AS1 levels. Patients with higher FOXD2-AS1 expression had a shorter survival time than those with lower expression (log-rank test; $\mathrm{P}=0.0070$ ). (C) FOXD2-AS1 expression was determined in HCC and normal liver cells. Data are presented as the mean \pm SD on the basis of at least 3 independent measurements. (D) FOXD2-AS1 expression in $140 \mathrm{HCC}$ tissue specimens and neighboring healthy liver tissues. ${ }^{* *} \mathrm{P}<0.01$.

FOXD2-AS1 enhances the viability and migration of HCC cells. To explore the involvement of FOXD2-AS1 in HCC pathogenesis, Hep3B cells were selected for FOXD2-AS1 overexpression, while SK-HEP1 and HCCLM3 were selected for FOXD2-AS1 silencing. The expression levels of FOXD2-AS1 were significantly higher in FOXD2-AS1-transfected cells than in their vector-transfected counterparts (Fig. 2A), whereas FOXD2-AS1 expression was lower in siFOXD2-AS1-transfected cells (Fig. 2C). Subsequently, a cell counting assay was conducted to examine the influence of FOXD2-AS1 on cancer cell viability. The MTT assay revealed that compared with that of the control cells, the growth of the FOXD2-AS1overexpressing Hep3B cells was notably enhanced (Fig. 2B), whereas the downregulation of FOXD2-AS1 by siRNA effectively inhibited the viability of SK-HEP1 and HCCLM3 cells (Fig. 2D).

Next, to determine whether FOXD2-AS1 alters cell motility, we studied cell migration using a wound healing assay and Transwell chamber assay after the transfection of FOXD2-AS1 or siFOXD2-AS1 into the indicated cells. The ectopic expression of FOXD2-AS1 markedly increased the migration of Hep3B cells (Fig. 2G, left upper image). To quantify this effect, we calculated the number of cells that migrated to the underside of the Transwell chambers following transfection with either the FOXD2-AS1-expressing vector or the control vector. The migration of FOXD2-AS1-transfected cells was significantly higher than that of the control vector-transfected cells ( $\mathrm{P}<0.05$; Fig. $2 \mathrm{G}$, left lower image), whereas the migration of siFOXD2-AS1-transfected cells was considerably lower than that of siRNA control (siCon)-transfected cells (Fig. 2G, right images). Similarly, in the wound healing assay, FOXD2-AS1 promoted the migration of Hep3B cells while siFOXD2-AS1 decreased the migration of SK-HEP1 and HCCLM3 cells (Figs. 2E and 2F). Overall, these results indicated that the overexpression of FOXD2-AS1 enhanced biological behaviors related to tumor progression in HCC cells in vitro.

FOXD2-AS1 functions as a molecular sponge of miR-206. Recently, FOXD2-AS1 has been reported to play a role in cancer development by acting as a ceRNA for miRNAs (13). To determine whether FOXD2-AS1 has an analogous role in HCC, we predicted miRNA binding sites using DIANA software, which is a miRNA target identification tool. We identified 10 typical miRNAs and their corresponding target sequences in FOXD2-AS1. The miRNA expression levels were determined in pcDNA-FOXD2-AS1-transfected Hep3B cells via qRT-PCR. Compared with their corresponding expression levels in pcDNA-NC-transfected cells, the miR-363, miR-520a, miR-940, and miR-206 expression levels displayed a considerable decrease in pcDNA-FOXD2-AS1-transfected Hep3B cells (data not shown).

miRNA acts by tethering to Ago2, an essential part of the RNA-induced silencing complex (RISC) that is vital to gene silencing. Furthermore, miRNA targets can be separated from the RISC by Ago2 coimmunoprecipitation (14). To determine whether FOXD2-AS1 associates with the RISC, we identified miR-363, miR-520a, miR-940, miR-206, and FOXD2-AS1 in the Ago2 pellet. As revealed in Fig. 3A, miR-520a, miR-363 and FOXD2-AS1 expression wasincreased $\sim 3$-fold, whereas miR-206 expression was increased by $>3$-fold. Therefore, FOXD2-AS1 may be associated with the dysregulation of miR-206. For further confirmation, we generated luciferase reporter vectors 

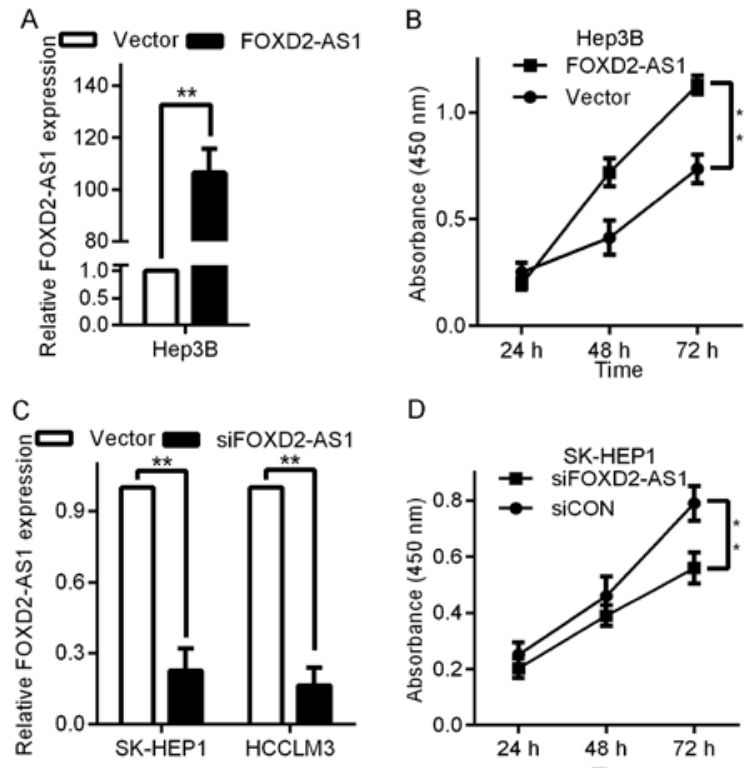

D
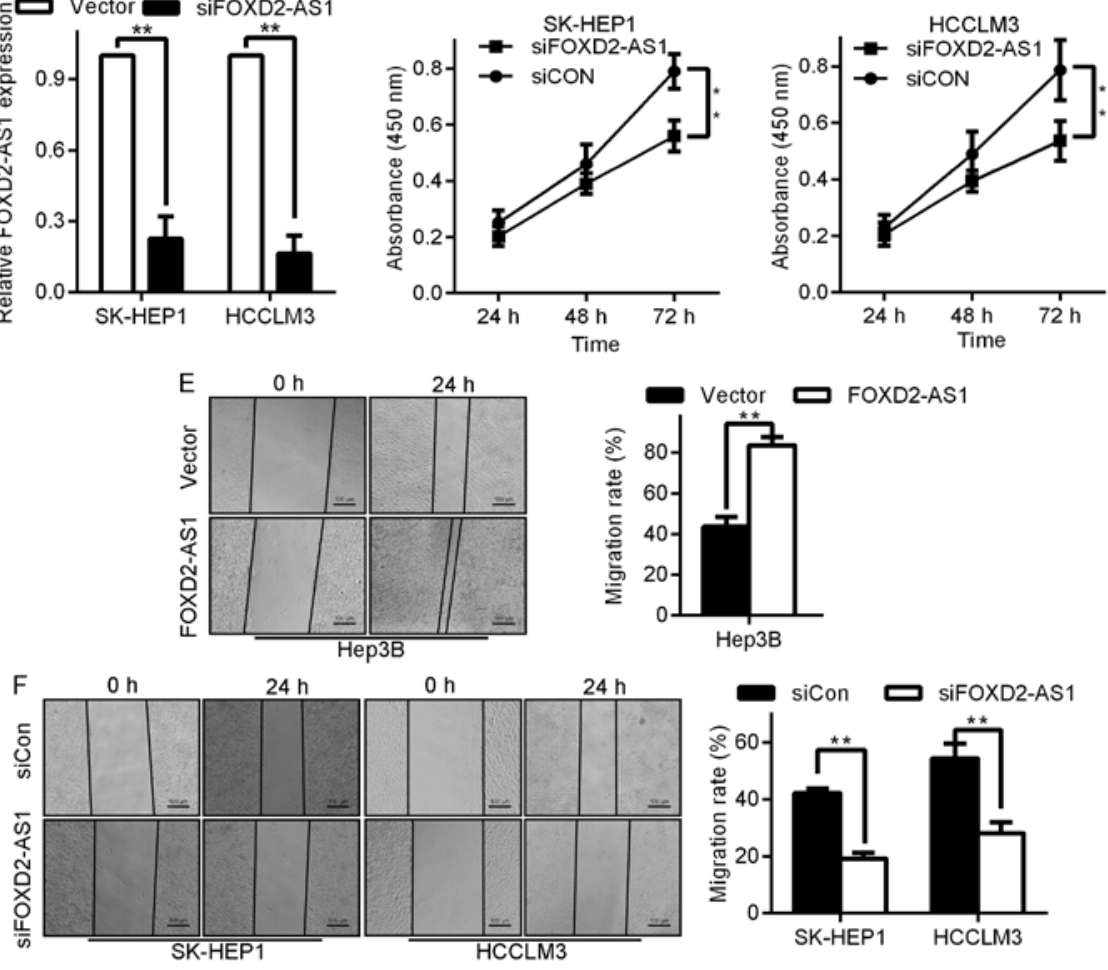

G
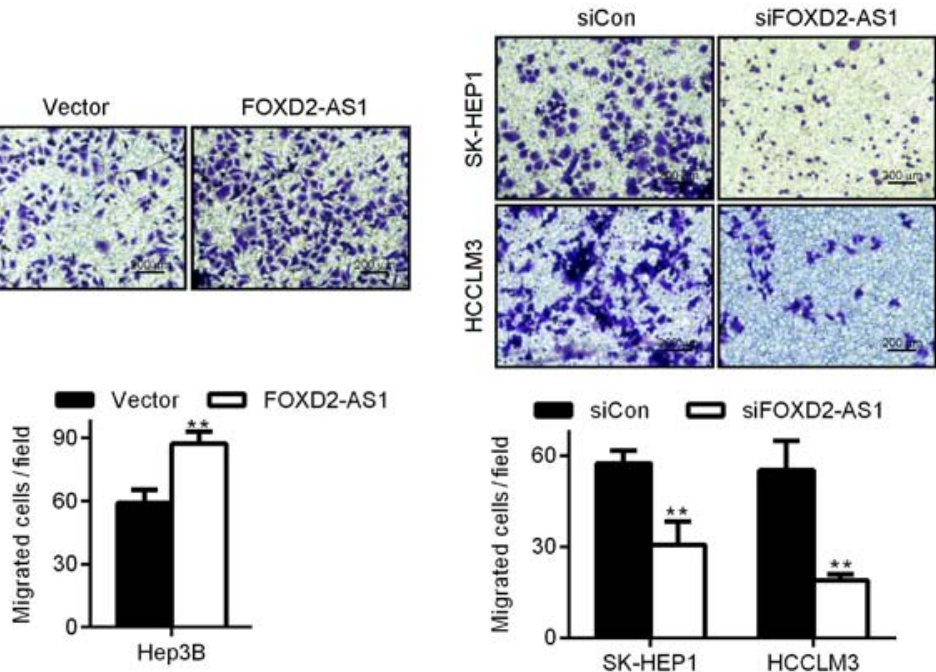

Figure 2. FOXD2-AS1 promotes the viability and metastasis of HCC cells. (A) FOXD2-AS1 overexpression in Hep3B cells was examined by qRT-PCR. (B) The upregulation of FOXD2-AS1 significantly promoted Hep3B cell viability as determined via MTT assay. (C) The knockdown of FOXD2-AS1 in SK-HEP1 and HCCLM3 cells was examined by qRT-PCR. (D) The downregulation of FOXD2-AS1 suppressed SK-HEP1 (left) and HCCLM3 (right) cell viability as illustrated via MTT assay. (E) Cell migration ability was evaluated via wound healing assay. Hep3B cells transfected with the control vector or FOXD2-AS1 expression vector were cultured to confluence, and a wound was created in the cell monolayer. After $24 \mathrm{~h}$, the relative wound closure was calculated as the ratio of the migration distance to the initial wound width at $0 \mathrm{~h}$. (F) The cell migration ability was evaluated via wound healing assay. SK-HEP1 and HCCLM3 cells transfected with siCon or siFOXD2-AS1 were cultured to confluence, and a wound was created in the cell monolayer. After $24 \mathrm{~h}$, the relative wound closure was calculated as the ratio of the migration distance to the initial wound width at $0 \mathrm{~h} .(\mathrm{G})$ Migration assays were conducted. Hep3B cells transfected with either the control vector or the FOXD2-AS1 expression vector and SK-HEP1 and HCCLM3 cells transfected with either siCon or siFOXD2-AS1 were seeded in Boyden chambers. The migrated cells were stained and counted in 5 arbitrarily chosen visual fields. Data are presented either as the mean \pm SD or as a representative graph/image from 3 independent measurements (paired t-test). ${ }^{* *} \mathrm{P}<0.01$. 


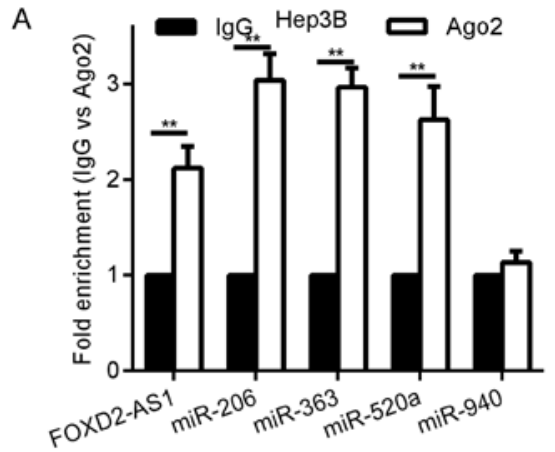

B
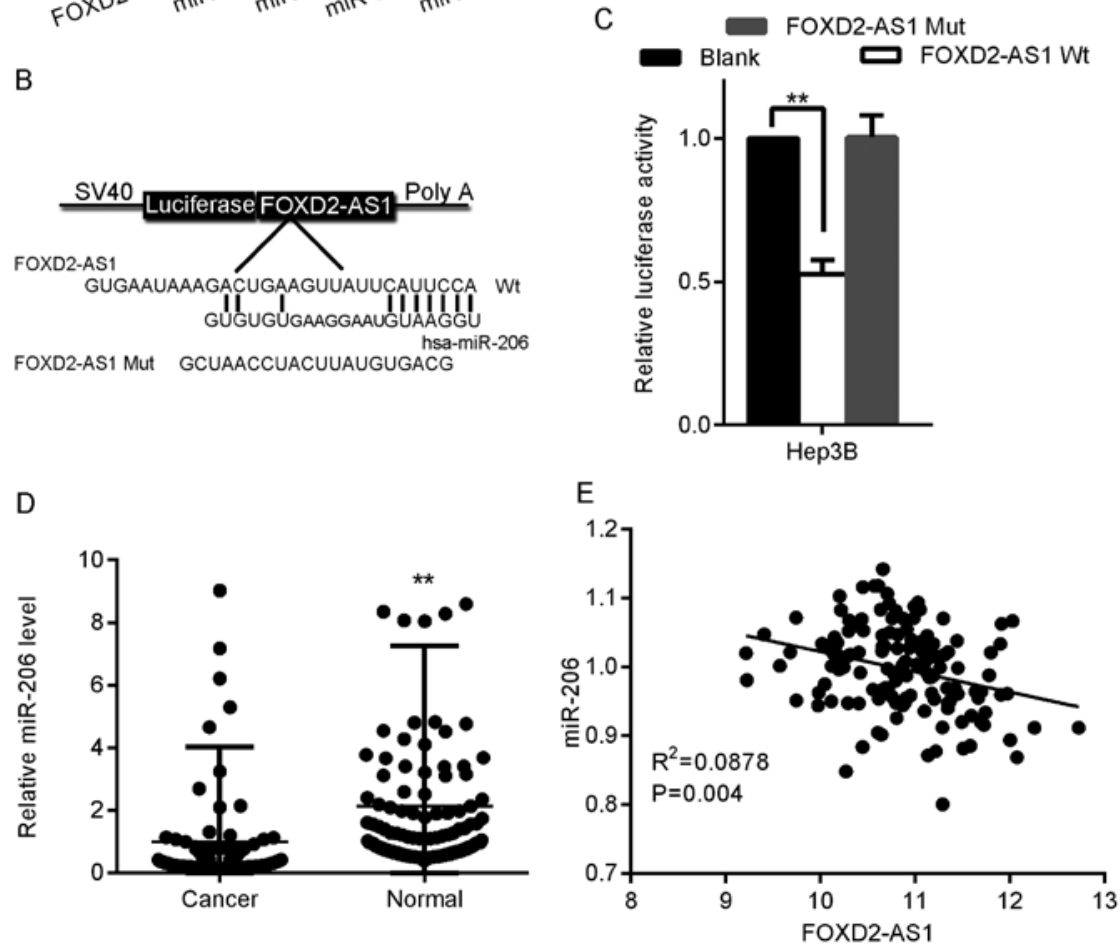

Figure 3. FOXD2-AS1 acts as a molecular sponge of miR-206. (A) The interaction between FOXD2-AS1, miRNAs and Ago2 was determined by examining Hep3B cell lysates via anti-Ago2 RIP. (B) The putative miR-206 target site on FOXD2-AS1 was identified. FOXD2-AS1 was mutated in the miR-206 binding site. (C) Hep3B cells were transfected with control, FOXD2-AS1-WT or FOXD2-AS1-Mut vectors, and luciferase activity was determined at $48 \mathrm{~h}$ via dual-luciferase reporter gene assay. (D) The expression of miR-206 in $120 \mathrm{HCC}$ tissues and the neighboring healthy liver tissues was determined. (E) The association between FOXD2-AS1 and miR-206 levels was determined in 120 HCC tissues via Spearman correlation analysis. FOXD2-AS1 and miRNA expression levels were estimated via qRT-PCR. ${ }^{* *} \mathrm{P}<0.01$.

expressing FOXD2-AS1 with either WT (FOXD2-AS1-WT) or mutant (FOXD2-AS1-Mut) miR-206 target sites (Fig. 3B). We established that the corresponding FOXD2-AS1-Mut construct did not suppress miR-206 expression (Fig. 3C), thus indicating that miR-206 is a FOXD2-AS1-specific miRNA. Subsequently, we quantified the expression levels of miR-206 in $120 \mathrm{HCC}$ tissues from the same set of patients described in Fig. 1D; miR-206 expression was notably lower in the HCC tissues than in the paired neighboring healthy liver tissues (Fig. 3D), and the miR-206 level was significantly negatively correlated with the FOXD2-AS1 level (Fig. 3E).

miR-206 reverses the growth-promoting effect of FOXD2-AS1 on HCC cells. To study the significance of miR-206 binding in the FOXD2-AS1-related promotion of HCC progression, we ectopically expressed miR-206 in stable FOXD2-AS1overexpressing Hep3B cells and analyzed cell viability via the MTT assay. The expression of miR-206 was detected by real time PCR (Fig. 4A). The overexpression of miR-206 diminished the viability-promoting activity of FOXD2-AS1 (Fig. 4B); this effect was also observed in Hep3B (Fig. 4C) cells in the Transwell assay. These results revealed that FOXD2-AS1 promoted tumor progression partly via competitive binding to miR-206.

FOXD2-AS1 regulates the expression of the endogenous miR-206 target ANXA2. miR-206 operates as a tumor suppressor in humans via the downregulation of ANXA2 (15). To establish whether FOXD2-AS1 regulates HCC progression by influencing miR-206 targets, we examined the impact of FOXD2-AS1 on ANXA2 expression. After transfection with the FOXD2-AS1 expression vector, the ANXA2 expression in Hep3B cells noticeably increased at the protein level but not at the mRNA level (Fig. 5A and B). Additionally, transfection with siFOXD2-AS1 clearly reduced ANXA2 expression in Hep3B cells at the protein level but not at the mRNA level (Fig. 5A and B). Furthermore, similar to the overexpression of miR-206 and FOXD2-AS1, the overexpression of ANXA2 


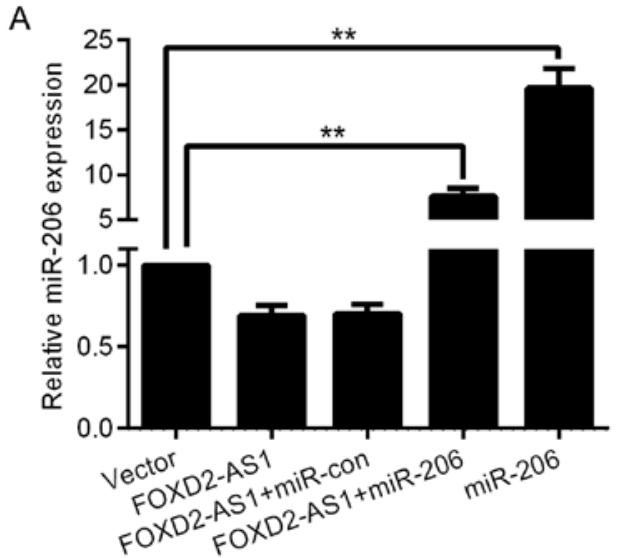

B

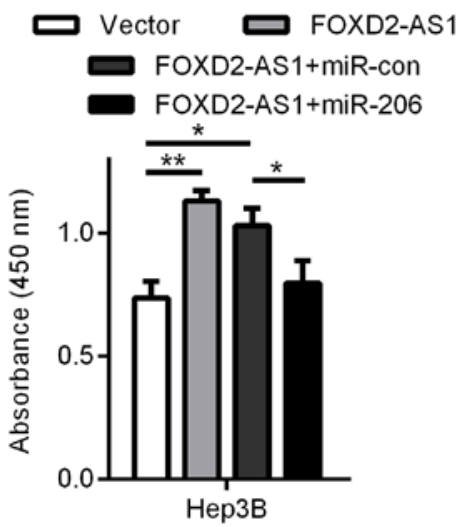

C

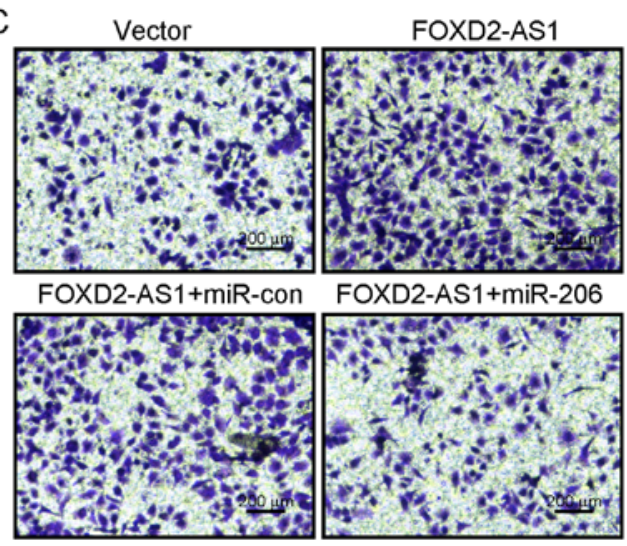

Figure 4. FOXD2-AS1 promotes HCC cell growth by competitive binding to miR-206. (A) The expression of miR-206 was detected by real time PCR (B) miR-206 mimics diminished the migration-promoting effect of FOXD2-AS1 on Hep3B cells. The number of cells was assessed by MTT assay. (C) miR-206 mimics diminished the migration-promoting effect of FOXD2-AS1 on Hep3B cells. All data are presented as the mean \pm SD on the basis of at least 3 independent measurements. ${ }^{*} \mathrm{P}<0.05,{ }^{* *} \mathrm{P}<0.01$.
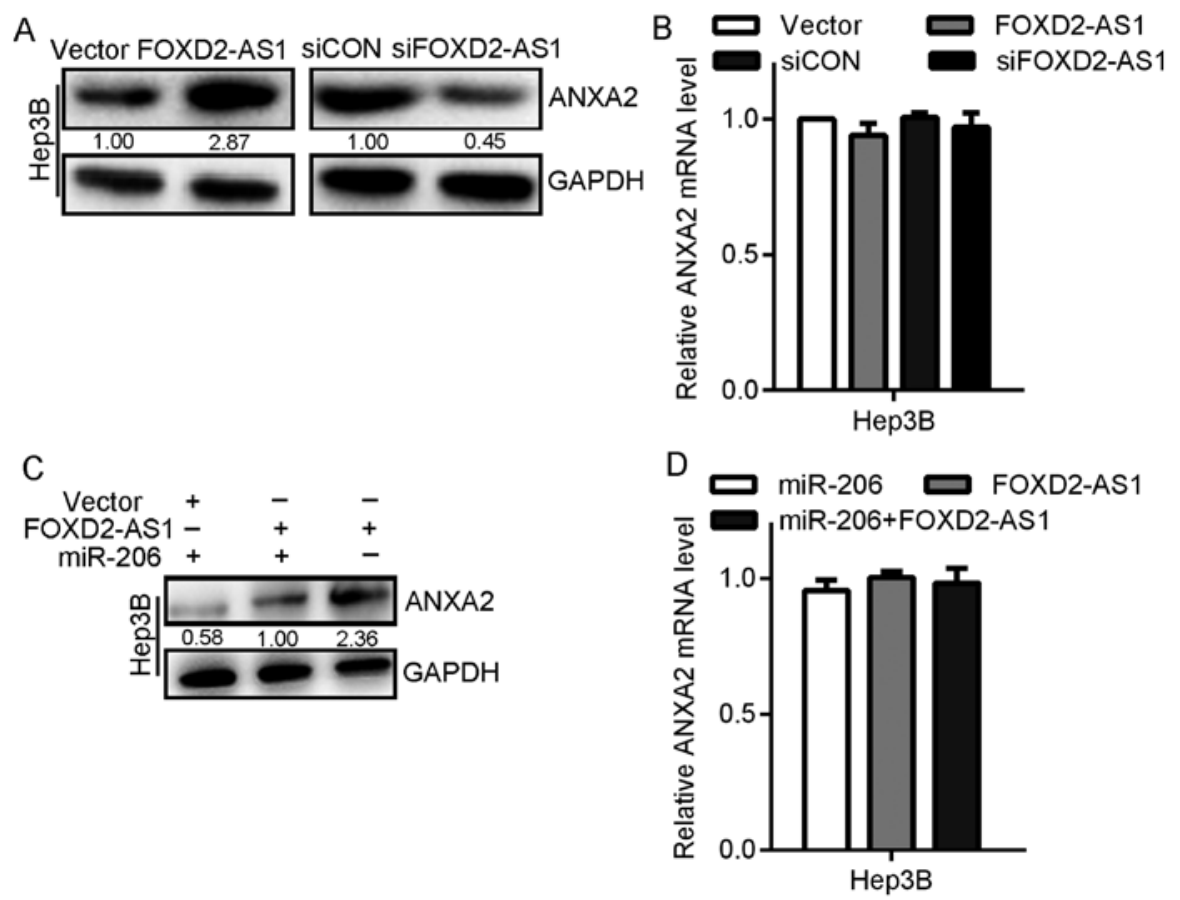

Figure 5. FOXD2-AS1 modulates the expression of the miR-206 target ANXA2. (A) After Hep3B cells were transfected with the FOXD2-AS1 expression vector or with siFOXD2-AS1, the ANXA2 protein levels were determined via western blotting. (B) After Hep3B cells were transfected with the FOXD2-AS1 expression vector or with siFOXD2-AS1, the ANXA2 mRNA levels were determined via qRT-PCR. (C) After Hep3B cells were transfected with the control vector, FOXD2-AS1, or FOXD2-AS1 combined with a miR-206 mimic, the ANXA2 protein levels were determined by western blotting. (D) After Hep3B cells were transfected with the control vector, FOXD2-AS1, or FOXD2-AS1 combined with a miR-206 mimic, the ANXA2 mRNA levels were examined by qRT-PCR. 
A

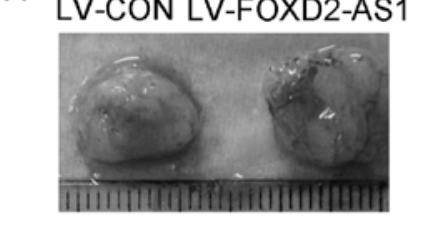

C

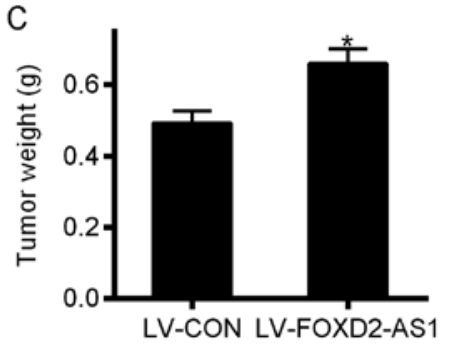

B

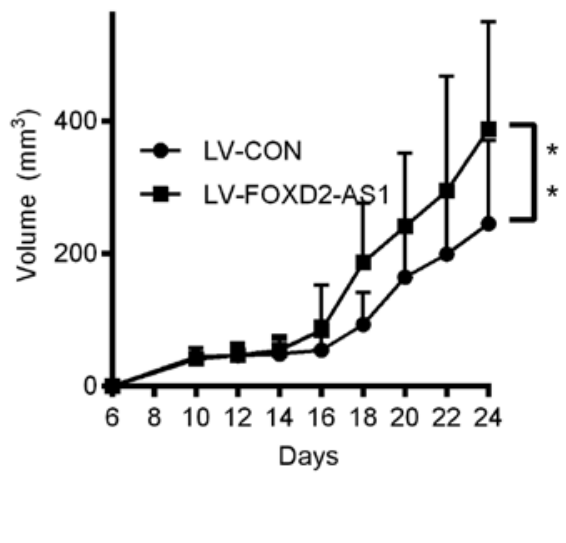

Figure 6. IncRNA FOXD2-AS1 promotes tumor growth in vivo. (A) Tumors were generated in the HCC xenograft nude mouse tumor model via the injection of lncRNA FOXD2-AS1-overexpressing or LV-CON HCC cells. (B) Tumor volumes in the FOXD2-AS1-overexpressing and LV-CON groups. ${ }^{* *} \mathrm{P}<0.01$. (C) Tumor weights in the FOXD2-AS1-overexpressing and LV-CON groups. " $\mathrm{P}<0.05$.

was detected in the Hep3B cell lines by western blotting but not by the measurement of the mRNA levels (Fig. 5C and D). Therefore, these results indicated that FOXD2-AS1 eliminated the suppression of ANXA2 induced by miR-206 and induced an oncogenic effect by modulating miR-206/ANXA2.

FOXD2-AS1 upregulation promotes HCC cell growth in vivo. FOXD2-AS1 promoted the viability and migration of HCC cells in vitro. To elucidate the impact of FOXD2-AS1 on tumor pathophysiology in vivo, HCC cells overexpressing FOXD2-AS1 or the appropriate control cells were injected into BALB/c nude mice. As displayed in Fig. 6A and B, after 24 days, the tumor growth in the FOXD2-AS1 xenograft group was considerably greater than that in the LV-CON xenograft group; the mean tumor volumes in the two groups were $387.45 \pm 161.41 \mathrm{~mm}^{3}$ and $245.34 \pm 125.64 \mathrm{~mm}^{3}$, respectively $(\mathrm{P}<0.01)$. After the 24 days, the mean tumor weights in the FOXD2-AS1 group were considerably higher than those in the LV-CON group (Fig. 6C).

\section{Discussion}

High-throughput RNA sequencing has revealed that approximately 70-90\% of human DNA is transcribed into RNA, but over $68 \%$ of the transcripts have been classified as ncRNAs $(16,17)$. Tens of thousands of lncRNAs, which are functionally defined as transcripts having a length of $>200$ nucleotides and no protein-coding capacity, exist; many of these are uniquely expressed in differentiated tissues or specific cancers (16). lncRNAs can exert either a negative or positive effect on gene expression via several pathways (18). Currently, the vital role of IncRNAs in the modulation of many pathophysiological processes, such as proliferation, development, cell cycle and apoptosis, has been recognized. In addition, lncRNAs play a crucial role in human tumors by functioning as either tumor suppressors or oncogenes (19). Hence, the elucidation of the principal lncRNA-related mechanisms underlying HCC generation and development is critical.

Significantly diverse IncRNA profiles may act as phenotypic signatures for the prognosis and treatment of multiple cancers. GPC3-AS1 is overexpressed in HCC due to a probable enhancement in histone acetylation at the promoter site; GPC3-AS1 overexpression results in the increased transcription of GPC 3 via PCAF and thus an enhanced level of cellular proliferation and metastasis (20). SNHG12 acts as a miR-199a/b-5p sponge; SNHG12 is upregulated in HCC, thereby stimulating growth and hindering apoptosis. Thus, the inhibitory effect of miR-199a/b-5p on MLK3 will be decreased, in turn upregulating MLK3 and its targets in the NF- $\mathrm{KB}$ signaling pathway (21). Via bioinformatics analysis combined with in vitro and in vivo functional experiments, FOXD2-AS1 was found to contribute to the progression of colorectal cancer by activating EMT and the Notch signaling pathway (22). In addition, lncRNA FOXD2-AS1 is considerably upregulated in non-small cell lung cancer (NSCLC). Loss- and gain-offunction assays revealed that FOXD2-AS1 enhanced NSCLC cell growth and tumor progression; furthermore, FOXD2-AS1 modulated Wnt/ $/$-catenin signaling in NSCLC cells (8). In the present study, we demonstrated that FOXD2-AS1 upregulation yielded an inferior clinical outcome in HCC patients; moreover, FOXD2-AS1 could be a potential biomarker for prognosis. Collectively, these results implied that clinical research on lncRNAs needs to be conducted and that additional investigations should be planned to identify additional lncRNAs as key prognostic molecular biomarkers and vital drug targets for HCC.

IncRNAs that act as ceRNAs or natural miRNA sponges are major post-transcriptional modulators of gene expression that compete with each other for binding to the same miRNAs $(23,24)$. For instance, lncRNA Unigene56159 enhanced metastasis by operating as a ceRNA for miR-140-5p in HCC (25). IncRNA HOTAIR acted as a ceRNA for miR-331-3p, thus regulating HER2 derepression and promoting tumor progression in gastric carcinoma (26). In the present study, we identified FOXD2-AS1 as an oncogenic player and illustrated a formerly unknown mechanism linking FOXD2-AS1 with miRNAs in HCC biology. By in vitro gain-and loss-of-function studies, FOXD2-AS1 was established to be associated with the miRNA-related regulatory network of HCC cell proliferation. Our observation that FOXD2-AS1 promoted cancer cell viability revealed a number of key aspects; for example, FOXD2-AS1 expression was extensively upregulated in HCC 
cells, thus indicating its prospective utility in HCC therapy. Accordingly, the overexpression of FOXD2-AS1 markedly promoted cell viability and migration in vitro. Moreover, the knockdown of FOXD2-AS1 negatively regulated cell migration. Subsequent mechanistic studies demonstrated that FOXD2-AS1 eliminated the inherent inhibitory activity of miR-206 on ANXA2; furthermore, the impact of FOXD2-AS1 on viability and migration was abrogated via the transfection of a miR-206 mimic. Further experiments confirmed that by competing for binding to miR-206, the ANXA2 3'-UTR acted as a ceRNA at the mRNA level in Hep3B cells, thus affecting the expression of ANXA2 at the protein level (Fig. 5). Therefore, targeting lncRNA-based signaling pathways may be a novel therapeutic strategy. However, the roles of lncRNAs in HCC carcinogenesis have not been thoroughly explored. A thorough investigation of the molecular mechanism underlying the initiation and progression of HCC is essential for facilitating the exploitation of novel therapeutic targets.

To this end, a recent study established that FOXD2-AS1 was linked to miR-363-5p and regulated the expression of the miR-363-5p target S100A, thus revealing that FOXD2-AS1 positively modulated post-transcriptional gene expression (13). The exploration of whether FOXD2-AS1 may perform its role in human tumors through interaction with miRNAs in addition to miR-363-5p would be of great interest.

In conclusion, this study indicated that FOXD2-AS1 expression could effectively predict the prognosis of HCC patients; however, this result requires confirmation in future investigations with a larger sample size. We employed mechanistic analysis to reveal the contribution of FOXD2-AS1 to the promotion of HCC progression by its function as a miR-206 sponge, and we illustrated a novel FOXD2-AS1/miR-206/ANXA2 signaling pathway regulatory network in HCC. These results indicated that FOXD2-AS1 is a key prognostic molecular biomarker as well as a vital drug target for HCC and that FOXD2-AS1 could contribute to the established crosstalk among conventional pathways.

\section{Acknowledgements}

Not applicable.

\section{Funding}

The present study was supported by the Scientific and Technological Project Foundation of Xi'an City [2017113SFYX007(6)] and by the Scientific and Fundamental Research Funds for the Central Universities of Xi'an Jiaotong University.

\section{Availability of data and materials}

The datasets used and/or analyzed during the current study are available from the corresponding author upon reasonable request.

\section{Authors' contributions}

YC, XL and LF conceived and designed the study. YC, JZ, $\mathrm{CZ}, \mathrm{GQ}$ and GW performed the experiments. YC, LF, SW and XC analyzed the data. YC and XL wrote the paper. LF,
SW and XC reviewed and edited the manuscript. All authors read and approved the manuscript and agree to be accountable for all aspects of the research in ensuring that the accuracy or integrity of any part of the work are appropriately investigated and resolved.

\section{Ethics approval and consent to participate}

The present study was approved by the Ethics Committee of the First Affiliated Hospital of Xi'an Jiaotong University (Xi'an, China). Prior to the the study, the patients signed written informed consent forms. All animal experiments were performed with stringent adherence to the Guide for the Care and Use of Laboratory Animals and were approved by the Laboratory Animal Care Committee of the Xi'an Jiaotong University (no. XJTULAC2018-462).

\section{Patient consent for publication}

Not applicable.

\section{Competing interests}

The authors declare that they have no competing interests.

\section{References}

1. Chen W, Zheng R, Baade PD, Zhang S, Zeng H, Bray F, Jemal A, $\mathrm{Yu}$ XQ and He J: Cancer statistics in China, 2015. CA Cancer J Clin 66: 115-132, 2016.

2. Morris KV and Mattick JS: The rise of regulatory RNA. Nat Rev Genet 15: 423-437, 2014.

3. Li S, Ma F, Jiang K, Shan H, Shi M and Chen B: Long non-coding RNA metastasis-associated lung adenocarcinoma transcript 1 promotes lung adenocarcinoma by directly interacting with specificity protein 1. Cancer Sci 109: 1346-1356, 2018

4. Cai T, Liu Y and Xiao J: Long noncoding RNA MALAT1 knockdown reverses chemoresistance to temozolomide via promoting microRNA-101 in glioblastoma. Cancer Med 7: 1404-1415, 2018.

5. Mondal T, Subhash S, Vaid R, Enroth S, Uday S, Reinius B, Mitra S, Mohammed A, James AR, Hoberg E, et al: MEG3 long noncoding RNA regulates the TGF- $\beta$ pathway genes through formation of RNA-DNA triplex structures. Nat Commun 6: 7743, 2015.

6. Benetatos L, Vartholomatos G and Hatzimichael E: MEG3 imprinted gene contribution in tumorigenesis. Int J Cancer 129: 773-779, 2011.

7. Yuan JH, Yang F, Wang F, Ma JZ, Guo YJ, Tao QF, Liu F, Pan W, Wang TT, Zhou CC, et al: A long noncoding RNA activated by TGF- $\beta$ promotes the invasion-metastasis cascade in hepatocellular carcinoma. Cancer Cell 25: 666-681, 2014.

8. Rong L, Zhao R and Lu J: Highly expressed long non-coding RNA FOXD2-AS1 promotes non-small cell lung cancer progression via Wnt $/ \beta$-catenin signaling. Biochem Biophys Res Commun 484: 586-591, 2017.

9. Tay Y, Rinn J and Pandolfi PP: The multilayered complexity of ceRNA crosstalk and competition. Nature 505: 344-352, 2014.

10. Tan J, Qiu K, Li M and Liang Y: Double-negative feedback loop between long non-coding RNA TUG1 and miR-145 promotes epithelial to mesenchymal transition and radioresistance in human bladder cancer cells. FEBS Lett 589 B: 3175-3181, 2015.

11. Chen Z,Xu D and Zhang T: Inhibition of proliferation and invasion of hepatocellular carcinoma cells by lncRNA-ASLNC02525 silencing and the mechanism. Int J Oncol 51: 851-858, 2017.

12. Livak KJ and Schmittgen TD: Analysis of relative gene expression data using real-time quantitative PCR and the 2(-Delta Delta C(T)) Method. Methods 25: 402-408, 2001.

13. Chen G, Sun W, Hua X, Zeng W and Yang L: Long non-coding RNA FOXD2-AS1 aggravates nasopharyngeal carcinoma carcinogenesis by modulating $\mathrm{miR}-363-5 \mathrm{p} / \mathrm{S} 100 \mathrm{~A} 1$ pathway. Gene 645: 76-84, 2018. 
14. Karginov FV, Conaco C, Xuan Z, Schmidt BH, Parker JS, Mandel $G$ and Hannon GJ: A biochemical approach to identifying microRNA targets. Proc Natl Acad Sci USA 104: 19291-19296, 2007.

15. Keklikoglou I, Hosaka K, Bender C, Bott A, Koerner C, Mitra D Will R, Woerner A, Muenstermann E, Wilhelm $\mathrm{H}$, et al: MicroRNA-206 functions as a pleiotropic modulator of cell proliferation, invasion and lymphangiogenesis in pancreatic adenocarcinoma by targeting ANXA2 and KRAS genes. Oncogene 34: 4867-4878, 2015.

16. Iyer MK, Niknafs YS, Malik R, Singhal U, Sahu A, Hosono Y, Barrette TR, Prensner JR, Evans JR, Zhao S, et al: The landscape of long noncoding RNAs in the human transcriptome. Nat Genet 47: 199-208, 2015.

17. ENCODE Project Consortium: An integrated encyclopedia of DNA elements in the human genome. Nature 489: 57-74, 2012.

18. Shi X, Sun M, Liu H, Yao Y and Song Y: Long non-coding RNAs: A new frontier in the study of human diseases. Cancer Lett 339: 159-166, 2013.

19. Kopp F and Mendell JT: Functional classification and experimental dissection of long noncoding RNAs. Cell 172: 393-407, 2018.

20. Zhu XT, Yuan JH,Zhu TT, Li YY and Cheng XY: Long noncoding RNA glypican 3 (GPC3) antisense transcript 1 promotes hepatocellular carcinoma progression via epigenetically activating GPC3. FEBS J 283: 3739-3754, 2016.
21. Lan T, Ma W, Hong Z, Wu L, Chen X and Yuan Y:Long non-coding RNA small nucleolar RNA host gene 12 (SNHG12) promotes tumorigenesis and metastasis by targeting miR-199a/b-5p in hepatocellular carcinoma. J Exp Clin Cancer Res 36: 11, 2017.

22. Yang $X$, Duan B and Zhou X: Long non-coding RNA FOXD2-AS1 functions as a tumor promoter in colorectal cancer by regulating EMT and Notch signaling pathway. Eur Rev Med Pharmacol Sci 21: 3586-3591, 2017.

23. Wang H, Huo X, Yang XR, He J, Cheng L, Wang N, Deng X, Jin $\mathrm{H}$, Wang $\mathrm{N}$, Wang $\mathrm{C}$, et al: STAT3-mediated upregulation of lncRNA HOXD-AS1 as a ceRNA facilitates liver cancer metastasis by regulating SOX4. Mol Cancer 16: 136, 2017.

24. Salmena L, Poliseno L, Tay Y, Kats L and Pandolfi PP: A ceRNA hypothesis: The Rosetta Stone of a hidden RNA language? Cell 146: 353-358, 2011

25. Lv J, Fan HX, Zhao XP, Lv P, Fan JY, Zhang Y, Liu M and Tang H: Long non-coding RNA Unigene 56159 promotes epithelialmesenchymal transition by acting as a ceRNA of miR-140-5p in hepatocellular carcinoma cells. Cancer Lett 382: 166-175, 2016.

26. Liu XH, Sun M, Nie FQ, Ge YB, Zhang EB, Yin DD, Kong R, Xia R, Lu KH, Li JH, et al: Lnc RNA HOTAIR functions as a competing endogenous RNA to regulate HER 2 expression by sponging miR-331-3p in gastric cancer. Mol Cancer 13: 92, 2014. 